\title{
Numerical determination of the material properties of porous dust cakes
}

\author{
D. Paszun ${ }^{\star}$ and C. Dominik
}

\begin{abstract}
Astronomical Institute "Anton Pannekoek", University of Amsterdam, Kruislaan 403, 1098 SJ Amsterdam, the Netherlands e-mail: dpaszun@science.uva.nl
\end{abstract}

Received 17 December 2007 / Accepted 12 February 2008

\begin{abstract}
The formation of planetesimals requires the growth of dust particles through collisions. Micron-sized particles must grow by many orders of magnitude in mass. To understand and model the processes during this growth, both the mechanical properties and the interaction cross sections of aggregates with surrounding gas must be well understood. Recent advances in experimental (laboratory) studies now provide the background for pushing numerical aggregate models to a new level. We present the calibration of a previously tested model of aggregate dynamics. We use plastic deformation of surface asperities as the physical model to match the velocities needed for sticking with experimental results. The modified code is then used to compute both the compression strength and the velocity of sound in the aggregate at different densities. We compare these predictions with experimental results and conclude that the new code is capable of studying the properties of small aggregates.
\end{abstract}

Key words. methods: numerical - planets and satellites: formation

\section{Introduction}

It is commonly accepted that planets form in protoplanetary disks. Giant planets are believed to be produced via one of two competing scenarios. The first one is a gravitational instability (Boss 2007). Clumps of gas (intermixed with dust) may become gravitationally bound and contract forming giant planets. This model is still being hotly debated (i.e. Pickett et al. 2000; Boley et al. 2006, 2007; Mayer et al. 2002, 2004, 2007). The second, more commonly accepted scenario is known as the core accretion model (Pollack et al. 1996). In this mechanism the solid core of a planet forms first and gas is accreted onto that core later. The core itself is believed to form by collisional accumulation of planetesimals. Because of their similarity to the giant planets' cores, terrestial planets must form out of planetesimals with the help of gravitational interactions.

The formation of planetesimals is also a subject of debate. The scenario initially proposed by Goldreich \& Ward (1973) assumed a laminar structure of the disk. When the dust sublayer reaches a critical thickness, a gravitational instability may form planetesimals from the dense and gravitationally bound concentrations of dust. However, this mechanism requires an extremely laminar nebula. The shearing motion of the dust layer causes a Kelvin-Helmholtz instability and therefore limits the thickness of the sublayer. The gravitational instability of the dust layer is then prevented. This was proved by Weidenschilling (1984), Cuzzi et al. (1993), and Johansen et al. (2006a).

The core accretion model requires micron-sized dust grains to grow into $\mathrm{km}$-sized planetesimals. These are over 27 orders of magnitude in mass. Radial drift alone may move dust particles inwards onto the central star before they reach a bigger size. The drift can move meter-sized particles all the way in within only 100 orbits (Weidenschilling 1977). Relative velocities of large

* Present address: Anton Pannekoek Institute, University of Amsterdam, Kruislaan 403, 1098 SJ Amsterdam, The Netherlands. bodies set another barrier against the accumulation of large particles (Weidenschilling \& Cuzzi 1993; Wurm et al. 2005a; Ormel \& Cuzzi 2007). A recent study by Johansen et al. (2006b) shows, however, that planetesimals might be produced by a gravitational collapse in the presence of turbulence. Meter-sized boulders concentrated in high-pressure turbulent eddies in the disk may form gravitationally bound clumps. Even if this process can really be made to work, dust particles must first grow over 18 orders of magnitude in mass to be able to form such dense accumulations. This growth must happen through the collisional sticking of dust particles.

The initially small, micron-sized, dust grains (also referred to later as monomers) collide and stick to each other thanks to attractive surface forces (Johnson et al. 1971). The fine dust is very well-coupled to the gas, meaning that the particles move collectively. The relative velocities are very small because of the Brownian motion (Brown 1828). These conditions lead to a quasi-monodisperse growth that tends to collide particles of similar sizes. The aggregates (further referred to as agglomerates or particles) formed in this case are fractal (Kempf et al. 1999; Blum et al. 2000; Krause \& Blum 2004; Paszun \& Dominik 2006).

The Brownian growth produces aggregates with a very open structure and the fractal dimension $D_{\mathrm{f}}=1.5$ (Blum et al. 2000; Krause \& Blum 2004; Paszun \& Dominik 2006). However, the gas density influences the growth. When the collisions are no longer ballistic due to high gas density and thus short stopping length, the fractal dimension decreases and, in the case of a very high gas density, it may even approach unity (Paszun \& Dominik 2006). This process may play a role in the innermost regions of protoplanetary disks.

The subsequent growth of the dust aggregates eventually leads to decoupling of the dust component from the gas. The particles start to be affected by turbulence, sedimentation, and radial drift (Weidenschilling 1977, 1980, 1984). The relative velocities 
thus increase, and collisions tend to occur between agglomerates of different sizes (Weidenschilling \& Cuzzi 1993). When the collision energy becomes higher than some threshold-restructuring energy, aggregates are compacted and their fractal dimension approaches $D_{\mathrm{f}}=3$ (Dominik \& Tielens 1997; Blum \& Wurm 2000). Further growth and compaction causes the particles to decouple from the gas more effectively and the relative velocities increase even more. Dominik \& Tielens (1997) and Blum \& Wurm (2000) have shown that the outcome of collisions can be categorized in terms of collision energy. Perfect sticking without restructuring occurs when the collision energy is lower than the threshold rolling energy, which is the energy needed to roll a single particle over an angle of $\pi / 2$. When the collision energy reaches this limit, the aggregates start to compress upon impact. The maximum compaction occurs when the collision energy equals the rolling energy times the number of contacts in the aggregates. The particles start to lose monomers, when the impact energy reaches the number of contacts in the aggregate times the energy needed to break one contact. Catastrophic disruption occurs when the energy reaches several times the number of contacts times the breaking energy. Therefore, as particles grow and decouple from the gas, eventually relative velocities are reached that will lead to shattering of the colliding aggregates (Ormel \& Cuzzi 2007).

This general picture has several missing elements. Although numerous experiments have been performed in different size and velocity regimes (Krause \& Blum 2004; Blum \& Wurm 2000; Wurm et al. 2005a,b; for a review see Blum \& Wurm, ARA\&A, 2008 , in press), our understanding of the processes involved in the growth of aggregates is still incomplete. The restructuring mechanism for instance is understood only qualitatively. The degree of the collisional compaction still remains a mystery. This, however, is crucial for the growth of the meter sized aggregates. The sticking efficiency as a function of the particle density along with the density evolution must be determined in order to understand quantitatively the growth of meter sized boulders.

The low strength of the aggregates due to the fractal structure leads to fragmentation in the case of fast impacts. The distribution of fragment sizes must be also determined as a function of the collision energy. This makes it possible to keep track of the realistic size distribution in the disk. Small particles, if not replenished in the disk, are very quickly swept up by larger grains (Dullemond \& Dominik 2005). Thus the small particles should be supplied to the disk by some processes. Dominik \& Dullemond (2008) show that the infall of grains from the parent cloud is rather unrealistic and requires fine tuning of parameters to reproduce observational data. Thus collisional fragmentation is the most likely mechanism that can explain the population of small grains in protoplanetary disks. However, long before fractal aggregates approach velocities high enough to cause fragmentation, they undergo collisional compression (Blum \& Wurm 2000; Weidenschilling \& Cuzzi 1993). Thus the only scenario leading to fragmentation of fractals is collision with much larger non-fractal particle.

The current understanding of processes like sticking, bouncing and fragmentation of aggregates is poor. The understanding of this processes on micro scales may fill these gaps and allow extrapolations to the larger sizes. This may be resolved by two approaches. Experiments may be performed in a laboratory to provide useful data. However, this way is available only for aggregates below centimeter in size. Larger particles are currently inaccessible for experiments. The second, theoretical approach, provides understanding of the material properties of porous matter and reach aggregates of meter size and beyond. Thus it would be ideal to provide theoretical predictions for centimeter sized and larger aggregates.

Sirono (2004) has developed a model capable of simulating meter-sized and larger aggregates, using smoothed particles hydrodynamics (SPH). In this case one particle in the model is an aggregate characterized by material properties, such as compressive strength, tensile strength, density, and sound speed. To obtain some of these properties, he fitted power-law functions to experimental data of compression and tensile strength. This method was then also used by Schäfer et al. (2007). Further studies require, however, a more precise determination of the material properties of porous bodies. These may be obtained in laboratory experiments or in computer simulations as presented in this work.

More realistic Solar nebula dust analogs were used in experiments by Blum et al. (2006). They investigated aggregates made of micron-sized dust particles. Moreover, different aggregates used in these experiments consisted of spherical or irregularly shaped monomers. The compression and tensile strength curves for these dust cakes were determined.

Although experiments quantitatively describe processes in $\mathrm{cm}$-sized aggregates, it is difficult to access for small aggregates of a few microns. Aggregate dynamics models (Dominik \& Tielens 1997; Dominik \& Nübold 2002) are ideal for simulations of these small-scale structures. This method spatially resolves single monomers, which is certainly needed to understand physics of the large meter-sized bodies. Until now the main drawback of this aggregate dynamics model has been missing quantitative agreement with experiments, even though the qualitative agreement has already been established (Blum \& Wurm 2000). Thus a calibration of this model is required.

Another aggregate dynamics model has recently been presented by Wada et al. (2007). They made use of potential energies to derive forces acting between grains at different degrees of freedom. In this case they only present a 2D case, but their results agree with findings of $N$-body model presented earlier by Dominik \& Tielens (1997). Wada et al. (ApJ, 2007, submitted) show the first results of compression and disruption of 3D aggregates in head - on collisions. Although the model qualitatively agrees with experiments, as is studied by Dominik \& Tielens (1997), the quantitative mismatch is still present. Wada et al. (2007) do not show any solution to the quantitative disagreement between theory and laboratory experiments. This paper addresses this issue and provides mechanisms that fit our model to the empirical data.

In this paper we present the calibration of the aggregates dynamics model developed by Dominik \& Tielens (1997) and Dominik \& Nübold (2002). We fit the code using experimental data and test it further. Modifications that are implemented in the code are presented, together with a few possible application of the model.

\section{The model}

To study the agglomeration mechanisms involved in the growth of planetesimals, we used the SAND code (soft aggregate numerical dynamics) developed by Dominik \& Nübold (2002). It is an $\mathrm{N}$-body model of a system of spherical particles interacting via surface forces.

Two monomers only feel the attractive force when they are in contact. The surfaces of the particles are deformed and form a contact area. When the particles are pulled outwards, increasing the relative distance, the contact area decreases and the 
monomers are pulled back to the equilibrium position by the surface forces. The compression of the system, on the other hand, leads to increasing the contact area and repulsive force pushing the particles apart. A detailed description of the surface forces was provided by Johnson et al. (1971) (referred to as JKR). The influence of adhesion forces on the contact between particles was also studied by Derjaguin et al. (1975). The model is able to treat long-range magnetic forces (Dominik \& Nübold 2002); however, these are not the subject of our current study.

There are two main processes that govern the events during a collision. The first one breaks a contact between two monomers. This dissipates part of the energy and weakens or destroys aggregates participating in the collision. The second process is a rolling motion of a monomer over another grain. This also dissipates energy and causes a restructuring of an aggregate. This restructuring may be attributed to either compression, which results in strengthening the aggregate, or decompression, i.e. weakening the aggregate's structure.

The JKR theory predicts a critical force that is needed to separate two particles. This prediction was tested for the case of micron-size Silica spheres by Heim et al. (1999). Two monomers were pulled off each other using an Atomic Force Microscope. The measured pull-off force agreed with the force predicted by the JKR theory. The pull-off force is

$F_{\mathrm{c}}=3 \pi \gamma R$,

where $\gamma$ is a surface energy and $R=\left(\frac{1}{R_{1}}+\frac{1}{R_{2}}\right)^{-1}$ is a reduced radius of the spheres in contact. Thus the results of the experiments confirm the theoretical predictions.

A similar experiment was performed to determine the horizontal forces acting on the particles in contact (first studied theoretically by Dominik \& Tielens 1997). The horizontal displacement of the contact zone causes a torque acting against the displacement. The resulting rolling friction was measured in laboratory experiments, again using an AFM (Heim et al. 1999).

\subsection{Rolling friction}

Rolling friction is one of the most important energy dissipation channels in the restructuring of aggregates (Dominik \& Tielens 1997). It is thus very importance to treat it in a correct way. The theoretical derivation by Dominik \& Tielens (1997) shows that the energy associated with initiating rolling is expressed as

$e_{\text {roll }}=6 \pi \gamma \xi_{\text {crit }}^{2}$

where $\xi_{\text {crit }}$ is a critical displacement at which the inelastic behavior occurs and energy is dissipated. Initially, $\xi_{\text {crit }}$ was assumed to be close to inter - atomic distances (Dominik \& Tielens 1997). The rolling motion causes a shift of the contact area, implying that the contact at one end has to be broken to form it at the other side of the contact area. The experimentally determined friction showed that the critical displacement must be larger. The determined value was $\xi_{\text {crit }}=3.2 \mathrm{~nm}$, meaning that the displacement is close to ten inter atomic distances. Dominik \& Nübold (2002) has already taken that into account and used the value of $\xi_{\text {crit }}=10 \AA$ in their model. This was applied, however, to different material than the one investigated in the lab. We followed Heim et al. (1999) and applied a higher value for $\xi_{\text {crit }}=20 \AA$, which is approximately 10 inter atomic distances.

\subsection{The pull-off force and the critical velocity for sticking}

The dominating mechanism that dissipates energy at low velocities is rolling. At higher impact speeds, however, other channels become more important. Chokshi et al. (1993) and Dominik \& Tielens (1997) calculated how much of the initial energy is dissipated in the collision between two particles. This gives the maximum energy at which the particles can stick in a head-on collision. The critical velocity is given by

$v_{\text {crit }}=1.07 \frac{\gamma^{5 / 6}}{E^{* 1 / 3} R^{5 / 6} \rho^{1 / 2}}$,

where $\gamma, R$ and $\rho$ are surface energy, reduced radius and mass density, respectively. The equation $E^{*-1}=\frac{1-v_{1}^{2}}{E_{1}}+\frac{1-v_{2}^{2}}{E_{2}}$ is a reduced elasticity modulus with $v_{\mathrm{i}}$ and $E_{\mathrm{i}}$ being Poisson's ratio and Young's modulus, respectively, of grain $i$. When Eq. (3) is applied to a $R_{1}=0.6 \mu \mathrm{m}$ silica grain, impacting flat silica surface, it gives the critical velocity $v_{\text {crit }}=0.18 \mathrm{~ms}^{-1}$. This was tested again in experiments. Poppe et al. (2000) show that such a particle can stick to the surface at significantly higher velocities on the order of $1 \mathrm{~ms}^{-1}$. The measured velocities were $1.2 \pm 0.1 \mathrm{~ms}^{-1}$ for $0.6 \mu \mathrm{m}$ grains and $1.9 \pm 0.4 \mathrm{~ms}^{-1}$ for $0.25 \mu \mathrm{m}$ grains. They used a slightly different definition of the critical velocity. The critical velocity was defined as the velocity at which the probabilities of sticking and bouncing are equal to $50 \%$. They measured the sticking velocity for different materials, shapes and sizes of particles. The resulting critical velocity was shown to depend on the size of the monomer as a power law with an index of about 0.53 . Although the theoretically derived slope $R^{-5 / 6}$ of the power law is different from the empirical data, they are still consistent within error bars. Moreover the power law was fitted to only two data points.

We assume that the theoretical dependence on the radius is correct and consistent with the experiment. The discrepancy between the absolute values of the critical velocity, however, has to be revised, and doing so is absolutely essential for meaningful results.

To better understand the mechanisms involved in the sticking of the monomers, we refer to experiments by Dahneke (1975) and Poppe et al. (2000). They investigated the bouncing of micron-sized spheres off a flat silica surface. In the earlier experiment, the impacting particle was made of soft polystyrene, while the later one used silica grains.

Dahneke (1975) experimentally determined the critical velocity to be about $1 \mathrm{~m} \mathrm{~s}^{-1}$. The ratio of the rebound velocity to the incident speed (coefficient of restitution) decreases in this case with decreasing impact velocity. At very high velocities of about $20 \mathrm{~m} \mathrm{~s}^{-1}$, Dahneke (1975) noticed a decrease in the restitution coefficient with increasing impact speed. He related that behavior to plastic deformation. In the experiment by Poppe et al. (2000) the first positive value of the coefficient of restitution indicates a critical velocity on the order of $\sim 1 \mathrm{~m} \mathrm{~s}^{-1}$.

To scale the model to the experimental data, we introduce a mechanism that dissipates part of the initial energy at the moment of first contact. The plastic deformation of small asperities on the surface of the grain is an easy way to dissipate the energy and increase the critical velocity. Below we estimate the central pressure in order to compare it to the yield strength of $104 \mathrm{MPa}$ (Callister 2000) for silica ${ }^{1}$. Chokshi et al. (1993), on the other hand, argue that the yield strength for very small bodies is nearly

\footnotetext{
1 Since the yield strength of brittle material like silica is not defined, flexular strength is given.
} 
0.2 times the Young's modulus of the material. This corresponds to $11 \mathrm{GPa}$ for silica.

The maximum pressure in the contact area occurs in the center of the contact. The radial distribution of pressure in the contact zone is (Dominik \& Tielens 1996)

$p(r, a)=6 \frac{F_{\mathrm{c}}}{\pi a_{0}^{2}} \frac{a}{a_{0}}\left(1-\left(\frac{r}{a}\right)^{2}\right)^{1 / 2}-2 \frac{F_{\mathrm{c}}}{\pi a_{0}^{2}}\left(\frac{a}{a_{0}}\right)^{-1 / 2}\left(1-\left(\frac{r}{a}\right)^{2}\right)^{-1 / 2}$,

where $a_{0}$ in this equation is the equilibrium contact radius given as

$a_{0}=\left(\frac{9 \pi \gamma R^{2}}{E^{*}}\right)^{1 / 3}$.

Now we need to estimate the maximum contact radius that is reached during the collision. The radius of the contact area increases with increasing normal load. The force applied to the sphere during the collision is $F=\mathrm{d}(m v) / \mathrm{d} t$, where $\mathrm{d}(m v)$ is the momentum of the impacting particle and $\mathrm{d} t \approx 10^{-9} \mathrm{~s}$ is the collision time. With this force applied, the contact radius is

$a=\left(\frac{3 R}{4 E^{*}}\left(F+6 \pi \gamma R+\sqrt{\left.(6 \pi \gamma R)^{2}+12 \pi \gamma R F\right)}\right)^{1 / 3}\right.$,

as derived by Johnson et al. (1971). Thus in the case of the velocity $v=1 \mathrm{~m} / \mathrm{s}$, the central pressure is close to $1 \mathrm{GPa}$. This pressure is, however, exactly in between the two values of the yield strength specified above, making plastic deformation possible. The lower limit of the yield strength $(104 \mathrm{MPa})$ is reached at radii $r / a<0.914$, which exposes $83 \%$ of the contact area to potential plastic deformation.

This idea was investigated before by Tsai et al. (1990). The energy consumption in such a deformation can be expressed as

$E_{\text {asp }}=Y V_{\text {asp }}$,

where $Y$ is the yield strength of the deforming material and $V_{\text {asp }}$ the volume of the asperities that are flattened during the collision. To calculate the dissipated energy we need to get the volume of asperities. We follow Tsai et al. (1990) again and calculate the maximum contact area given by

$a_{\max }=\left(\left(\frac{3 R}{4 E^{*}}\right)\left[F_{\mathrm{eq}}+6 \pi \gamma R+\sqrt{(6 \pi \gamma R)^{2}+12 \pi \gamma R F_{\mathrm{eq}}}\right]\right)^{1 / 3}$

(Chokshi et al. 1993) and the equivalent impact force given by Tsai et al. (1990) as

$F_{\text {eq }}=2.5^{3 / 5} E_{\text {kin }}^{3 / 5} R^{1 / 5} E^{* 2 / 5}$.

Now we can get the volume of the deformed material by multiplying the contact area by the volume of a single asperity and the number of asperities per surface area

$V_{\text {asp }}=\frac{2}{3} \pi r_{\text {asp }}^{3} N_{\text {asp }} * \pi a_{\text {max }}^{2}$,

where $r_{\text {asp }}$ is the radius of a single asperity. Here we assume the bumps to be hemispheres distributed homogeneously over the surface of a particle or a target. In our case we use a parameter that describes the total volume plastically deformed per unit of the surface area. This way we have only one parameter that defines how efficiently the energy is dissipated. In reality the pressure in the contact area is compressive in the center and tensile on the edge. Thus our parameter may be slightly higher

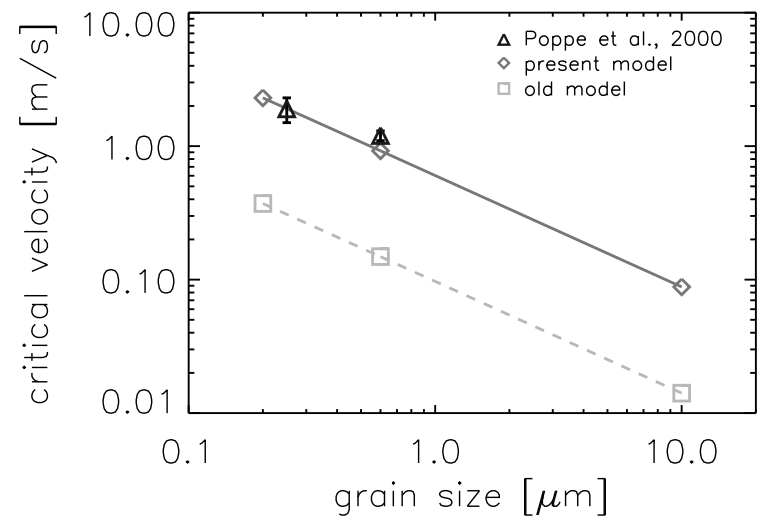

Fig. 1. Critical velocity as a function of a grain size. Squares indicate the model without additional dissipation process, diamonds show the present model with the energy dissipation by plastic deformation of surface roughness and triangles show experimentally determined values by Poppe et al. (2000) with error bars.

than what we present. Figure 1 shows the critical velocities determined experimentally (Poppe et al. 2000) for two different grain sizes. The critical velocity obtained using our model is also plotted showing that we have successfully fitted our model and that we can reproduce the experimental results.

The volume of the asperities deformed upon collision is very small in our model. When we assume surface roughness to be hemispheres with radii $r_{\text {asp }}=1 \mathrm{~nm}$, the fraction of the area occupied by the asperities is only about $2 \%$. We can thus still say that the molecular size asperities may be responsible for increasing the critical velocity. Moreover, we see in Fig. 1 that the dependence of the sticking velocity on the grain size is not affected, and the only difference is that the energy regime has been shifted to the level measured in experiments.

To avoid confusion, we must again stress that we do not claim that the plastic dissipation takes place during the collisions of silica spheres. We simply implement additional scaling parameter in order to fit our model to the experimental data. The discrepancy between the empirical data and theory should be further investigated. We also think that plastic deformation might need some more attention, because of the very high pressure that is present in the center of the contact area and the relatively low strength of the material considered in this work.

\subsection{Excitation and cooling}

In parallel to the energy dissipation due to contact breaking, energy can be dissipated via other channels in the vertical degree of freedom (along the line connecting centers of two grains in contact). Two grains held together by the surface force vibrate relative to each other (Dominik \& Tielens 1995). Ideally the oscillation is frictionless so no dissipation occurs. In reality, however, the vibration causes an oscillation in the size of the contact area. When decreasing the contact size, part of the energy is dissipated by breaking the connections at the edges of the contact area. This ultimately leads to a cooling of the aggregate and damps the vibrations. If this mechanism is not taken into account, successive slow collisions may heat up the aggregate and ultimately lead to "evaporation" of monomers from the aggregate surface.

In fact we observed this phenomenon in our simulations of linear chains. Successive collisions of grains with an aggregate caused an increase in the amplitude of the oscillation and eventually led to several contacts being broken. All individual collision 
velocities were far below the sticking velocity, proving that this mechanism may produce artificial results.

To resolve this problem, we introduced a weak damping force, which was intended to slowly dissipate the vibrational energy. The force acts in the vertical direction with respect to the contact area. The damping force is expressed as

$F_{\text {damp }}=$ const. $v_{z}$,

where $v_{z}$ is the vertical component of the relative velocity, and const is arbitrarily chosen to damp exponentially 95 percent of the vibration energy within $\sim 100$ oscillation periods. By tuning the constant to this value, we made certain that the influence of the damping force is not significant within short timescales of a few first vibration periods, where the sticking or bouncing event is determined. The dissipation takes place in the first period and, if the particle does not lose enough energy, it will bounce. If the damping force is too strong, it may remove enough energy to allow sticking. We made sure that the main energy dissipation process that sets the critical velocity is the plastic deformation mechanism so that the sticking velocity is not affected by the presence of the damping force.

To show the energy leakage due to the damping force, we calculated the total energy in a vibrating pair of monomers. The particles were displaced from the equilibrium position and the kinetic and potential energies calculated. In the case of a frictionless oscillation, the potential energy is

$E_{\mathrm{p}}=\int_{0}^{\delta} F\left(\delta^{\prime}\right) \mathrm{d} \delta^{\prime}$,

where $\delta$ is a displacement such that the distance between centers of the two grains is $r_{1}+r_{2}-\delta$. The vertical force

$F(a)=4 F_{\mathrm{c}}\left(\left(\frac{a}{a_{0}}\right)^{3}-\left(\frac{a}{a_{0}}\right)^{3 / 2}\right)$

depends on the contact size $a$ and equilibrium contact radius $a_{0}$. The integral (12) may then be changed into

$E_{\mathrm{p}}=\int_{0}^{a} F\left(a^{\prime}\right) \mathrm{d} a^{\prime}$

To get the energy, we solve Eq. (14) by changing the variable from $\delta$ to $a$ using the relation

$\delta=\frac{1}{2} \frac{a_{0}^{2}}{R}\left(2\left(\frac{a}{a_{0}}\right)^{2}-\frac{4}{3}\left(\frac{a}{a_{0}}\right)^{1 / 2}\right)$.

The potential energy is then given as

$E_{\mathrm{p}}=4 F_{\mathrm{c}} \frac{a_{0}^{2}}{R}\left[\frac{2}{5}\left(\frac{a}{a_{0}}\right)^{5}-\frac{2}{3}\left(\frac{a}{a_{0}}\right)^{7 / 2}+\frac{1}{6}\left(\frac{a}{a_{0}}\right)^{2}\right]$.

The kinetic energy is simpler. We just add kinetic energies of all monomers together,

$E_{\mathrm{k}}=\sum_{i} \frac{m_{i} v_{i}^{2}}{2}$,

where $m_{i}$ and $v_{i}$ are the mass and absolute velocity of the $i$ th grain.

The total energy $E_{\text {tot }}$ in the case of a frictionless oscillation is plotted in Fig. 2a. To better see variations in the kinetic energy, we shifted it to the level of the potential energy. The shift is equal to the potential energy of the system in the equilibrium
$E_{\mathrm{p}}=\int_{0}^{\delta_{0}} F\left(\delta^{\prime}\right) \mathrm{d} \delta^{\prime}$. The total energy in this case is conserved and the amplitude constant. When we enable the damping force, the energy starts to leak. The potential and kinetic energies may be calculated using the same formulas. The energy dissipation is presented in Fig. 2b.

The energy loss due to the damping force is very weak and removes about $95 \%$ of the total energy within approximately 100 vibration periods. Thus other processes, such as rolling and breaking, dominate the energy dissipation. In particular the critical velocity for sticking is not influenced at all by this damping force, because the assumed plastic deformation of asperities dissipates nearly the entire collision energy within the first vibration period.

\section{Applications}

The presented model is a very good tool that can be used to study the aggregation of dust particles. However, the modifications that we introduced in the previous section need further verification. Although the critical velocity is well-fitted to the experiments, it is very useful to test the model more against laboratory experiments. When the model has been successfully verified, it can be applied confidently to the study of aggregates dynamics. Compaction and fragmentation of dust aggregates are processes that require detailed investigation (Dullemond \& Dominik 2005). Our model is perfectly suited to this task. We can provide in depth understanding of micro-physics that governs compaction and fragmentation on this scale. Phenomena involving aggregates of $\mathrm{mm}$ and larger sizes require an entirely different approach. They must be treated with different methods i.e. smoothed particles hydrodynamics (SPH). However, to do this, material properties, such as compressive strength and sound speed, are needed. Below we present simulations that can be directly compared to experiments and provide good tests of our model. We simulate compression of a dust cake and determine its compressive strength. We also determine the sound speed in these porous aggregates.

\subsection{Compression}

Blum \& Schräpler (2004) measured the compressive strength of dust cakes in the laboratory. The sample was prepared by random ballistic deposition (RBD). Single monomers were shot from one direction at low velocity (hit-and-stick) and then grew the dust agglomerate. The resulting "cake" was about $2 \mathrm{~cm}$ in diameter and similar in height. The finished cake was later placed between two flat surfaces. The load was applied to the upper surface causing it to move towards the lower surface compressing the dust sample. To simulate compression of a dust cake, we need to prepare the proper setup.

\subsubsection{Setup}

In the experiment by Blum \& Schräpler (2004), the applied pressure resulted in compression of the dust cake. Measurement of the cake volume resulted in determination of a filling factor $\phi$.

Our setup was organized in a similar manner to the experimental one. Our code, however, can only handle spherical particles. Thus instead of two planes, we used two very big "wall" grains, each with a diameter of $2 \times 10^{-2} \mathrm{~cm}$. The sample was shaped as a cylinder, and the distance between two compressing "wall" grains was adjusted to fit the micro dust cake exactly. 


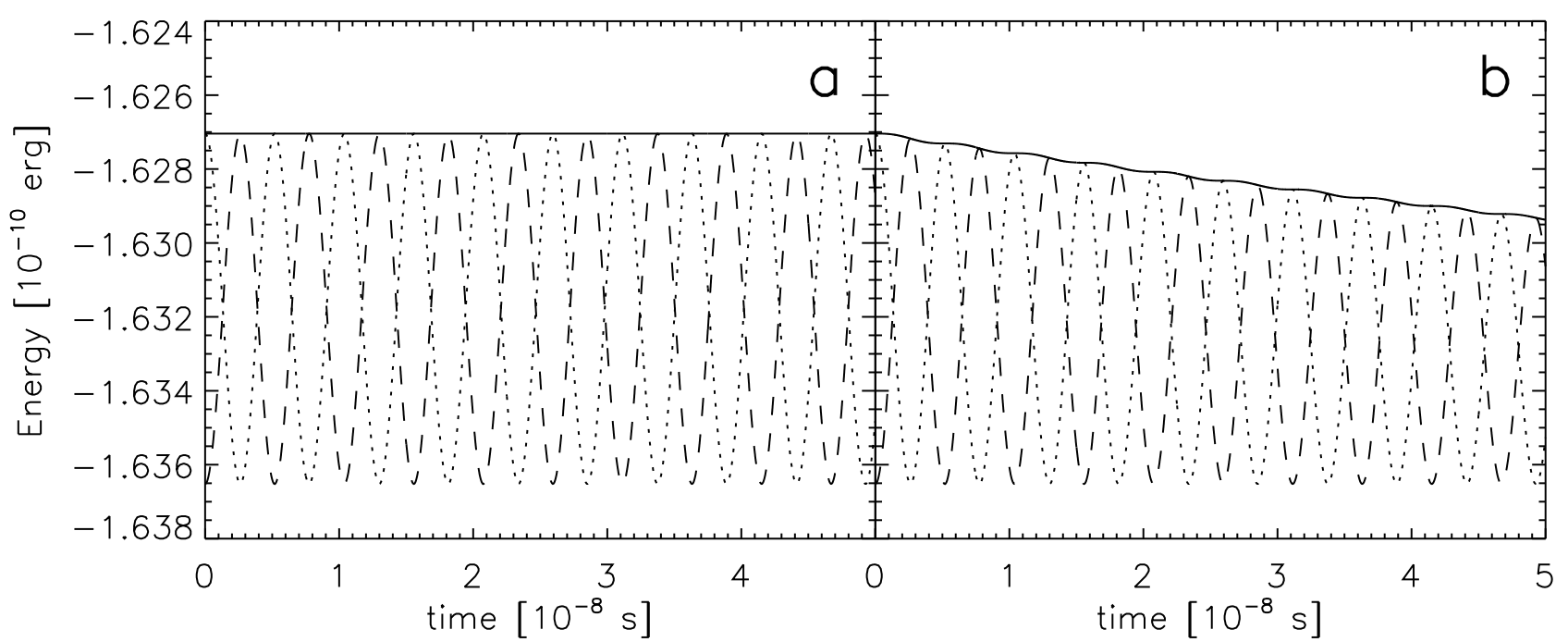

Fig. 2. The total energy is redistributed between the kinetic energy (dashed line) and the potential energy (dotted line). At the maximum and minimum separations the potential energy is maximum, while the kinetic energy is at its highest in the equilibrium position. The kinetic energy was shifted down for a better overview (see text). No damping case - a), and the energy leak form the system due to the damping force - b).

Our dust cake was grown via the particle cluster aggregation method (PCA). The target aggregate (initially a monomer) was randomly oriented before each collision with a grain approaching at a random impact parameter. Any successful hit resulted in perfect sticking without any restructuring. The new target was then randomly oriented, and a new grain was shot at it again. The resulting aggregate was then shaped into a cylinder by removing all grains outside of the desired contour.

The size of the cake was $13.2 \mu \mathrm{m}$ in diameter and it was $13.2 \mu \mathrm{m}$ high. When filled by 291 monomers with a radius of $0.6 \mu \mathrm{m}$, the filling factor of the cake was $\phi=0.146$. For comparison the dust cake used in the real experiment had the filling factor $\phi=0.15 \pm 0.01$. The monomer size in that experiment was similar, $r_{0}=0.75 \mu \mathrm{m}$.

The compression was done by moving one of the large grain surfaces at constant velocity. While it was approaching, the second grain was fixed in its position and was unable to move even with extreme pressures. The sample was thus compressed by one surface against the other one. The initial setup is presented in Fig. 3a. Two large grains on both sides of the aggregate are the back wall, fixed plane on the right and the approaching, compressing plane on the left. The aggregate is placed in between and the particles can escape sideways, this way increasing the diameter of the cake.

To simulate quasi static compression, we fixed the velocity of the compressing "wall" grain to $0.05 \mathrm{~m} / \mathrm{s}$. This is over an order of magnitude lower than a critical velocity and much lower than the sound speed in this medium (see Sect. 3.2). Thus the assumption that we are in a quasi static regime is reasonable. The dominant acceleration of a single monomer, in this case, is caused by surface forces.

For the purpose of this compression simulation, we disabled the net force acting onto the compressing "wall" particle, but we saved the record of this force for each time step. Thus the approaching surface cannot be stopped and moves with constant speed. For each time step the net force was stored and later used to determine a pressure.

At each successive step, the dust cake becomes slightly more compressed. The degree of compression can be related to the force that was needed to get the cake into this state.

\subsubsection{Results}

The small size of the dust cake used in this numerical experiment causes a low number of contacts of the compressed aggregate with the approaching surface. Consequently, the net force applied to the compressing surface is strongly variable. Each new contact formed between the dust cake and the incoming surface results in a sudden decrease in the force. The new connection is initially stretched and thus the surface is attracted. Similarly, oscillations of monomers at the surface cause additional variation. This makes it more difficult to uniquely determine the compression force. To overcome this problem, for ten each successive time steps we choose the one with the highest force, because ultimately this is the force required to compress the cake. The pressure is calculated as $P=\frac{F}{S}$, where $F$ is the normal load and $S$ the cross-section area.

Figure 3 shows the initial setup and the results of compression with increasing pressure. The lowest pressure cannot restructure the aggregate. The height of the dust cake is almost unchanged. A higher pressure of $2 \mathrm{kPa}$ compresses the cake. The monomers on the cake's surface are pushed down into the cake. At a pressure of $1 \times 10^{4} \mathrm{~Pa}$, the cake is compressed significantly, causing a horizontal flow of the particles and thus an increase of the cake diameter. The evolution of the dust cake cross-section is shown in Fig. 4. The relative increase is affected by the size of the dust cake. What may be a negligible boundary effect in a large macroscopic aggregate, here it has a strong impact on the entire dust cake. When the diameter of the cake increases just by a diameter of a single monomer, the area increases by $40 \%$. In the experiment by Blum \& Schräpler (2004), a cake of about $2 \mathrm{~cm}$ diameter was compressed and the final increase in the cross-section was measured to be larger by a factor of 1.6 relative to the initial cross-section. In our simulation the area increased almost 4 times, subject to significant uncertainties in determining the dust cake cross-section. The initially cylindrical shape changed upon compression into an irregular profile.

To determine the volume filling factor, we only used the inner part of the cake. A cylindrical volume initially enclosing the dust aggregate, and used to determine the filling factor, only decreased in height. In this way we reduced the uncertainty that arose from the boundary effects. The initially porous 

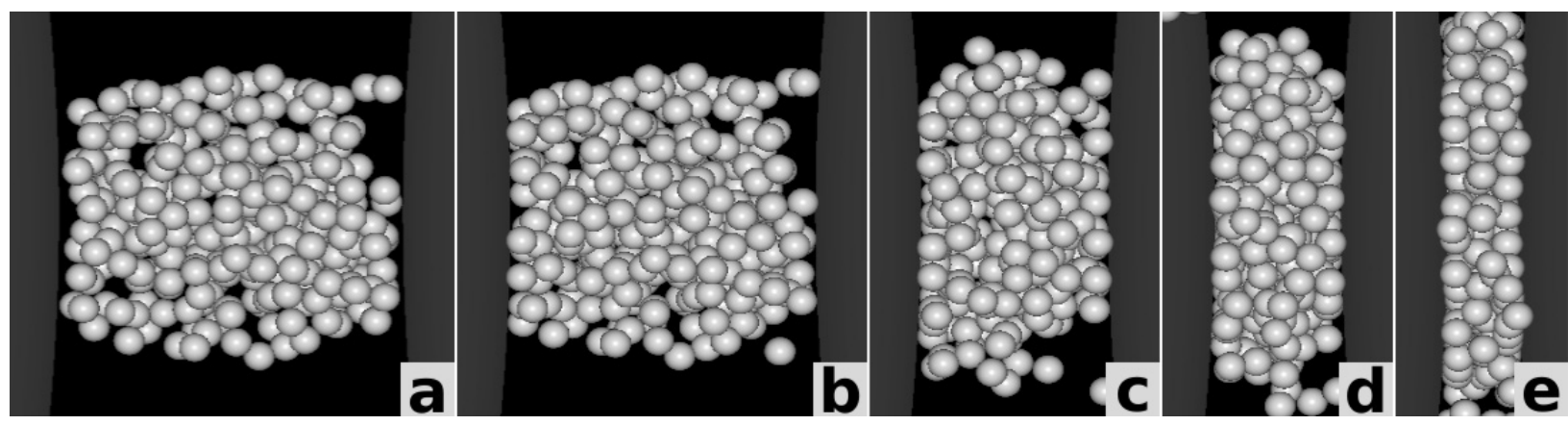

Fig. 3. The setup of the experiment. The dust cake in the center is compressed with different pressure. Initial arrangement (a), results of compression at $2 \times 10^{2} \mathrm{~Pa}(\mathbf{b}), 2 \times 10^{3} \mathrm{~Pa}(\mathbf{c}), 5 \times 10^{3} \mathrm{~Pa}(\mathbf{d}), 1 \times 10^{4} \mathrm{~Pa}(\mathbf{e})$.

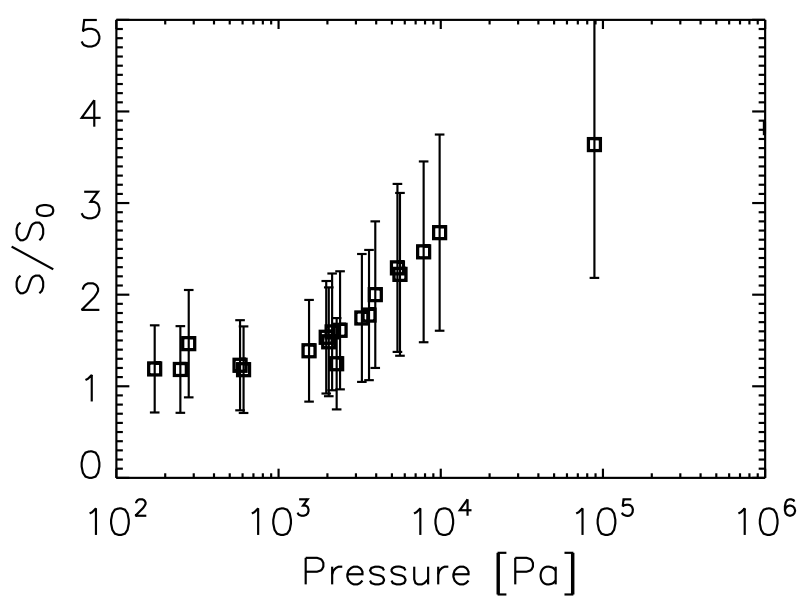

Fig. 4. The ratio of the cross-section of the dust cake to the initial crosssection as a function of the applied compressive pressure. The data presented here is from a single experiment. Each point is plotted with a $40 \%$ uncertainty in determination of the crosssection (see text).

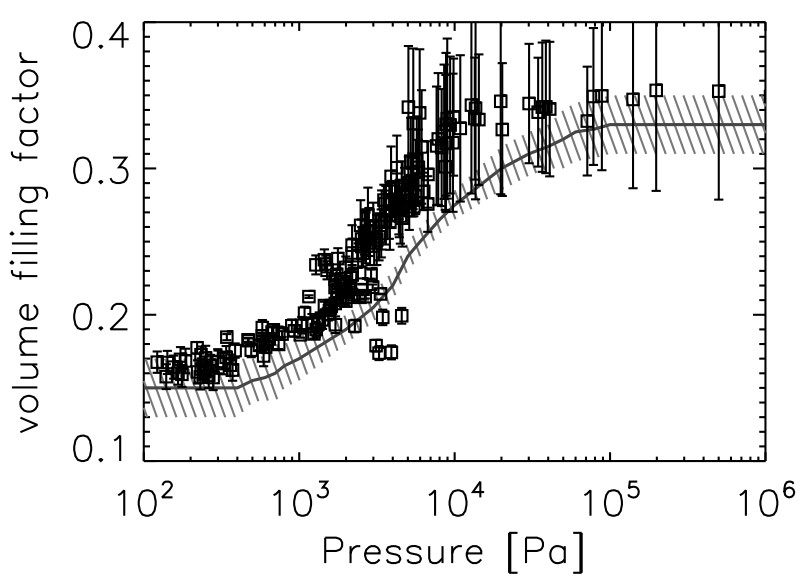

Fig. 5. The volume-filling factor vs. normal pressure. The solid line indicates the results of the laboratory experiment by Blum \& Schräpler (2004). The dashed area is the error to that data. Squares show the results of our single simulation. Error bars are due to difficulty determining of the volume of the final aggregate.

aggregate is deformed and can expand. Particles are pushed into the cake, filling voids. Thus the filling factor must increase as an effect of compression. Figure 5 shows our results, together with the data obtained in the laboratory experiments (Blum \& Schräpler 2004). Low pressures are unable to affect the aggregate. However, the boundary effects also cause problems. Initially the "wall" particle connects to the cake by only one monomer. This causes very strong variations in pressure. We show the compression curve starting at the point where the "wall" particle has made 5 contacts with the cake. At this point, however, a few grains are already pressed into the dust cake, causing an increase in the volume-filling factor. Thus our compression curve in Fig. 5 is shifted upwards. The filling factor is also overestimated by using big spherical grains as the compressing surfaces. The height of the dust cake we used is calculated as $D-2 R_{\text {compress, }}$, where $D$ is distance between centers of the two big grains, and $R_{\text {compress }}$ is their radius. Thus the volume occupied by the dust cake is actually slightly larger. This effect is initially less important, as it contributes only about a $2 \%$ error. However, it gets more relevant at greater pressure, where the cross-section of the dust cake is larger and the volume smaller.

Error estimation was done using the central parts of the dust cake, where boundary effects are smaller. The filling factor was determined by calculating the volume of monomers enclosed in a cylindrical volume. The error was then estimated to be the difference between the filling factors determined for two different cylinders. The first one had a radius of $6 \mu \mathrm{m}$, while the second one was one monomer radius smaller.

The volume filing factor is initially constant, until the pressure reaches a value of about $5 \times 10^{2} \mathrm{~Pa}$. The filling factor increases until it reaches the maximum compression of about $\phi=0.35$ and remains constant. The most interesting result is the resemblance of our findings to the laboratory experiments. The onset of compression fits the experimental data of Blum \& Schräpler (2004). Our compression curve follows the laboratory data tightly ending up at a similar value of the filling factor. The small differences between two curves are most likely due to the small size of our simulated dust cake.

\subsection{Sound speed}

One of the very important properties in the porous material is the sound speed. It must be lower than the bulk sound speed because the mass is being moved by a force acting on a very small contact area. The collision of two grains with supersonic velocities can result in complete disruption of those bodies. We first derive an analytical formula for the sound speed. For this we apply the JKR theory and assume that the signal transported is a very small perturbation. The main assumption is that the two monomers in contact behave like a perfect spring, which is the case for small amplitudes. However, the contact forces are asymmetric with respect to the equilibrium position. Thus compression of two particles results in different forces than stretching it to the same displacement. We can therefore expect that large amplitudes lead to modified sound speeds. In the following sections, we present an 
analytical approach and later show our simulations for both the simplified case of a linear chain of monomers and the general case of a non fractal aggregate.

\subsubsection{Analytical solution}

Each two monomers that are in contact are held together by surface forces acting in the contact area (Johnson et al. 1971). Mutual attraction inevitably leads to a vibrational spring-like motion, which in linear approximation can be written as

$F=-k\left(\delta_{0}-\delta\right)$,

where $\delta, \delta_{0}, k$, and $F$ are displacements, equilibrium displacement, spring constant, and restoring force, respectively. Note that the displacement $\delta$ is defined in a way that it increases, when the distance between two monomers is decreasing. In our case we want to determine the spring constant, which is needed to find the sound speed. Johnson et al. (1971) showed that the surface force in the contact area is related to the contact area radius $a$ by

$F=4 F_{\mathrm{c}}\left(\left(\frac{a}{a_{0}}\right)^{3}-\left(\frac{a}{a_{0}}\right)^{3 / 2}\right)$.

We also know the relation between the displacement and contact radius

$\delta=6^{1 / 3} \delta_{\mathrm{c}}\left(2 a_{0}^{-2} a^{2}-4 / 3 a_{0}^{-1 / 2} a^{1 / 2}\right)$,

where $\delta_{\mathrm{c}}=1 / 2 \frac{a_{0}^{2}}{6^{1 / 3} R}$ is a critical displacement for breaking the contact. With these equations we can determine the spring constant and later the sound speed. First we differentiate Eqs. (19) and (20) at $a=a_{0}$ to get

$\left.\frac{\mathrm{d} F}{\mathrm{~d} a}\right|_{a=a_{0}}=\frac{6 F_{\mathrm{c}}}{a_{0}}$

and

$\left.\frac{\mathrm{d} \delta}{\mathrm{d} a}\right|_{a=a_{0}}=\frac{5 \delta_{0}}{a_{0}}$

where $\delta_{0}=\frac{a_{0}^{2}}{3 R}$ is an equilibrium displacement. Now we can write

$\frac{\mathrm{d} F}{\mathrm{~d} \delta}=\frac{\mathrm{d} F}{\mathrm{~d} a} \frac{\mathrm{d} a}{\mathrm{~d} \delta}=\frac{\mathrm{d} F}{\mathrm{~d} a}\left(\frac{\mathrm{d} \delta}{\mathrm{d} a}\right)^{-1}$

and substituting Eqs. (21) and (22), we get

$\mathrm{d} F=\frac{6}{5}\left(9 \pi \gamma R^{2} E^{\star 2}\right)^{1 / 3} \mathrm{~d} \delta$.

Since the restoring force is exactly opposite, we may add the minus sign here and see that the spring constant $k$ is

$k=\frac{6}{5}\left(9 \pi \gamma R^{2} E^{\star 2}\right)^{1 / 3}$.

With the spring constant of the oscillating system of two spheres in contact, we can proceed to the calculation of the sound velocity. For a spring the velocity of sound is given by

$c_{\mathrm{s}}=\sqrt{\frac{k L}{\rho_{\mathrm{l}}}}$,

where $L$ is the total length of the spring (or set of the springs) and $\rho_{1}$ a mass of the spring per unit length. We can then determine
Table 1. Material properties used in this work for silica.

\begin{tabular}{ccc}
\hline \hline$E^{*}\left[\mathrm{dyn} / \mathrm{cm}^{2}\right]$ & $\gamma\left[\mathrm{erg} / \mathrm{cm}^{2}\right]$ & $\rho_{0}\left[\mathrm{~g} / \mathrm{cm}^{3}\right]$ \\
\hline $2.78 \times 10^{11}$ & 25.0 & 2.65 \\
\hline
\end{tabular}

the velocity in a linear chain of $n$ grains in contact, each $r_{0}$ in radius. The length of such a string of grains is

$L=n\left(2 r_{0}-\delta_{0}\right)+\delta_{0}-2 r_{0}$.

Since the spring constant for a set of springs is simply $k_{L}=k / n$, the sound velocity can be written as

$c_{\mathrm{s}}=\sqrt{\frac{k_{L} L}{\rho_{1}}}=\sqrt{\frac{k L^{2} / n}{n m_{0}}}=\frac{L}{n} \sqrt{\frac{k}{4 / 3 \pi r_{0}^{3} \rho_{0}}}$.

Substituting Eqs. (25) and (27), we get

$c_{\mathrm{s}}=\left(1-\frac{1}{n}\right)\left(2 r_{0}-\delta_{0}\right) \sqrt{\frac{\left(9 \pi \gamma R^{2} E^{\star 2}\right)^{1 / 3} 6 / 5}{4 \pi r_{0}^{3} \rho_{0} / 3}}$.

Now we can try to see how the sound velocity in such system depends on the size of a single monomer:

$c_{\mathrm{s}} \propto r_{0} \sqrt{\frac{r_{0}^{2 / 3}}{r_{0}^{3}}}=r_{0}^{-1 / 6}$.

This means that to double the speed of sound we need to use grains that are 64 times smaller. Therefore, the sound speed in this case is a very weak function of monomer size.

The sound speed of an infinitely long chain of $0.6 \mu \mathrm{m}$ silica grains is $c_{\mathrm{s}}=513 \mathrm{~m} / \mathrm{s}$. If we apply our findings to a chain of 50, 0.6 micron sized, silica grains, the sound velocity turns out to be $c_{\mathrm{s}}=503 \mathrm{~m} / \mathrm{s}$. We can compare it now to the previous results obtained in research of granular medium composed of $\mathrm{mm}$ sized and bigger grains. In all our simulations and analytical calculations, we used material properties as specified in Table 1.

Hascoët et al. (1999) has developed a model of macroscopic grains to study the propagation of sound in a granular chain. The centimeter-sized grains they use do not interact in this case via attractive surface forces. The signal is transported due to the Hertzian stress that arises as an effect of overlap of grains in contact. They also apply the spring theory but with an arbitrarily chosen spring constant $k$. For values of $k$ in the range between $k=10^{6} \mathrm{~N} / \mathrm{m}$ and $k=10^{8} \mathrm{~N} / \mathrm{m}$ and mass density of $1.9 \times 10^{3} \mathrm{~kg} / \mathrm{m}^{3}$, the sound velocity they derive is in the range $300 \mathrm{~m} / \mathrm{s}$ to $3000 \mathrm{~m} / \mathrm{s}$. Similar results were obtained by Mouraille et al. (2006). A sound speed $c_{\mathrm{s}} \approx 200 \mathrm{~m} / \mathrm{s}$ was found for closely packed grains. In this case monomer radius was $1 \mathrm{~mm}$, density $2 \times 10^{3} \mathrm{~kg} / \mathrm{m}^{3}$, and the spring constant $k=10^{5} \mathrm{~N} / \mathrm{m}$.

\subsubsection{Numerical experiment}

We performed a numerical experiment to verify our findings. We prepared a linear chain of 50 monomers. The first one in line was slightly displaced from its equilibrium position. When the simulation started, the grain started to move to reach its equilibrium and the second grain was disturbed. This motion propagated until it finally reached the last grain. The total distance traveled by the density wave was $99\left(r_{0}-\delta_{0}\right)$. For 50 silica monomers with radii $r_{0}=0.6 \mu \mathrm{m}$, the travel time took $t=1.16 \times 10^{-7} \mathrm{~s}$, which results in the sound speed of $c_{\mathrm{s}}=512 \mathrm{~m} / \mathrm{s}$. We can now 


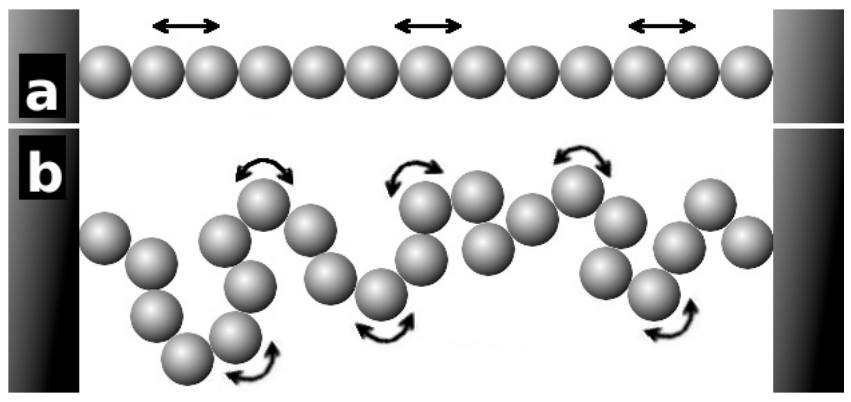

Fig. 6. Sketch of a sound wave propagation in a linear aggregate (a) and in a porous aggregate (b).

compare the value with the theoretically derived sound speed $c_{\mathrm{s}}=503 \mathrm{~m} / \mathrm{s}$. The difference between the theoretical velocity and the one that was obtained numerically is only about $3 \%$. Moreover in the simulation, the first grain is displaced by a finite distance meaning that the assumption of low displacements may not be entirely correct. The displacement of about $0.5 \delta_{0}$ is relatively large. We performed a series of simulations with stronger perturbations and the data obtained shows that the sound velocity increases as the perturbation strength increases.

\subsubsection{Porous aggregates}

Since a linear chain of monomers is a special case, we now discuss the more general agglomerates of irregular shape. The sound speed in such a system is affected by several things. First, the path length that a signal has to travel is not a straight line, so with a given speed, longer distances result in lower effective sound speed. For a small RBD aggregate (approximately 300 monomers), the effective distance was greater by only a factor of 1.5, thus the structure of an aggregate has a limited impact on the sound velocity by increasing the path length. The second factor that may have an effect is the tangential force. In a linear aggregate, the signal is passed forward by the presence of the vertical force, and the tangential force has no effect. However, grains interact also via rolling and sliding in irregular aggregates. This might lower the contribution of the vertical force and in this way change the sound speed. Figure 6 shows a sketch of how a sound wave propagates in different aggregates. Indeed linear aggregates involve only stronger, vertical forces and thus the signal travels faster. Irregular particles also make use of the tangential, rolling force.

To estimate the sound speed due to the rolling friction, we again used the spring theory. Dominik \& Tielens (1995) gives the recipe for the rolling friction as

$F_{\text {roll }}=6 \pi \gamma \xi$,

where the $\xi$ is a displacement from the equilibrium position. This force can be then expressed as

$F_{\text {roll }}=k \xi$,

with $k=6 \pi \gamma$ as a spring constant. We then apply this in Eq. (28) to get

$c_{\mathrm{s}}=\left(1-\frac{1}{n}\right)\left(2 r_{0}-\delta_{0}\right) \sqrt{\frac{6 \pi \gamma}{4 / 3 \pi r_{0}^{3} \rho_{0}}}$.

The dependence of the sound speed on a grain size, $c_{\mathrm{s}} \propto r_{0}^{-1 / 2}$, is in this case much stronger than in the case of the vertical force $\left(c_{\mathrm{S}} \propto r_{0}^{-1 / 6}\right)$.
When we apply Eq. (33) to the $1.2 \mu \mathrm{m}$ silica grains, we get a sound speed of $c_{\mathrm{s}}=16.5 \mathrm{~m} / \mathrm{s}$. This is a factor of 30 lower than the velocity derived for the linear chain. This suggests that the sound velocity in a porous aggregate might be significantly different from the speed derived for a linear chain of grains.

Sirono (2004) derived compressive and tensile strengths dependent on density and based on experimental data. Those relations were later used to develop an SPH model of large, mmsized, and larger aggregates collisions. The sound speed in an aggregate made of $0.1 \mu \mathrm{m}$ ice monomers was calculated to be $c_{\mathrm{s}}=\sqrt{E / \rho}$. He used values for the Young's modulus and the density to be $E=6 \times 10^{5} \mathrm{~Pa}$ and $\rho=0.1 \mathrm{~g} / \mathrm{cm}^{3}$, respectively. The resulting sound speed is then $c_{\mathrm{s}}=77.5 \mathrm{~m} / \mathrm{s}$.

When we apply our formula for the sound speed to 100 aligned $0.1 \mu \mathrm{m}$ ice grains, we get a sound speed of $c_{\mathrm{s}}=885 \mathrm{~m} / \mathrm{s}$. This is almost an order of magnitude higher. We have to keep in mind, however, that the speed in a linear chain may be considerably different to the one in a real porous aggregate. In the rolling case, the theoretical sound speed is also high with $c_{\mathrm{s}}=250 \mathrm{~m} / \mathrm{s}$.

Teiser (2007) performed a laboratory experiment, where he measured the sound speed in an RBD aggregate with filling factor $\phi=0.15$ and $1.5 \mu \mathrm{m}$ silica monomers. He hit the dust cake from below and measured the response at the surface with a force sensor. The measured velocity was $c_{\mathrm{s}}=30 \pm 4 \mathrm{~m} / \mathrm{s}$, very consistent with our results when the signal is assumed to be transferred through the rolling degree of freedom.

To numerically derive the sound speed and further test our model, we performed a simulation of an RBD dust cake. The cake was being pushed from one side, and we determined the response time of different particles in the aggregate. We combined the positions of the particles with their response time, which results in an average sound speed in the dust cake. Figure 7a shows the result of our simulations. For each monomer in the dust cake, we plotted a corresponding sound speed. The initially wide spread in the data shows that very linear structures are present inside the cake. This leads to a few particles with very fast sound speed. At greater distances, however, the range of sound speeds is much narrower showing that a signal is transported mainly via a rolling degree of freedom. The average sound speed that is determined at the far end of the dust cake is only a factor of about 1.5 higher than the sound velocity in the rolling degree of freedom.

We applied two different forces to the cake and later determined mean sound speeds in the cake for both cases. When a stronger force of $5 \times 10^{-6} \mathrm{~N}$ was applied to the cake, the sound speed reached $c_{\mathrm{s}} \approx 60 \mathrm{~m} / \mathrm{s}$, while the sound velocity was $c_{\mathrm{s}} \approx 20 \mathrm{~m} / \mathrm{s}$ with the lower force of $5 \times 10^{-8} \mathrm{~N}$. For one monomer, the limiting sound velocity was overcome, as calculated for linear chain of monomers. This, however, happened in the case of stronger force, and thus the low displacement approximation was most likely violated, leading to higher sound speeds.

The simulations show that the sound propagation is dominated by the rolling friction. To verify this, we ran another simulation meant to determine the sound speed in the aggregate with monomers arranged according to the cubic close packing (CCP) because this arrangement disables any rolling motion. The inset in Fig. $7 b$ shows the aggregate. We applied a force on one side of the agglomerate and determined the response time of different particles. Data in Fig. 7b shows that indeed closely-packed aggregates are characterized by much higher velocities than the porous ones. The monomers in each layer received the signal at a different time because the compressing planes were simulated by big grains, and thus central particles were hit first and 


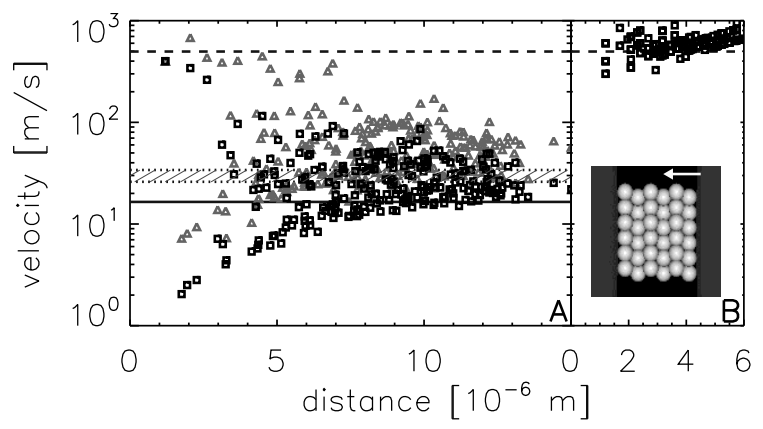

Fig. 7. Sound velocity in $\mathrm{m} / \mathrm{s}$ versus distance from the plane that hits the aggregate. Squares indicate an applied force of $5 \times 10^{-8} \mathrm{~N}$ and triangles $5 \times 10^{-6} \mathrm{~N}$. The left panel a) shows the results of sound speed determination in a RBD aggregate, while the right one b) in a CCP aggregate. The inset image shows the CCP aggregate placed in between two planes. The lines indicate sound velocities determined theoretically using vertical force in a linear chain of monomers (dashed line) and using rolling friction (solid line). The dashed area limited by dotted lines indicates the sound-speed range determined experimentally by Teiser (2007).

forwarded the signal. Thus particles at the edges of the aggregate responded later than the ones in the center of each layer. The velocity $c_{\mathrm{s}} \approx 600 \mathrm{~m} / \mathrm{s}$ shows that indeed sound speed in a porous medium depends strongly on porosity because of the forces involved in the transport of the signal and the longer path for the signal to travel in more porous aggregates. Using a constant sound speed in SPH simulations is bound to lead to spectacularly wrong results.

\section{Conclusions}

We have modified the original code by Dominik \& Tielens (1997) and Dominik \& Nübold (2002) and shown that a numerical N-particle method for studying the properties of aggregates can be calibrated to experimental results by including the flattening of small asperities on the surface of the grains and by using critical displacements for rolling grains consistent with measured values. The new code reproduces the measured critical velocities for sticking very well. We would like to emphasize that there is currently no proof of plastic deformation actually happening on the grain surfaces, but it works very well as a model.

We went on to measure the compressive strength of the aggregates and compared the results with experiments. We get very similar results with a small offset in porosity, which is most likely caused by the relatively small aggregates used in our simulations. Finally, we computed the sound velocity in an adhesiondominated porous material and showed that this leads to very interesting results. Three very different velocities play a role in such a medium, and the different velocities are separated by factors of about 10 . The fastest speed is the bulk material sound speed that only plays a role after a body has melted and rehardened. The second speed, a factor of $\sim 10$ lower, is the speed at which a signal is transported in a longitudinal wave in a linear chain. This speed applies either in a perfectly linear chain or in an aggregate that has been compacted enough that rolling of grains is no longer possible. Finally, the slowest speed is the one transmitted by rolling forces in a crooked chain of grains. A small decrease stems from the longer path the sound has to take in a porous aggregate. By far the largest fraction stems from the weak forces in the rolling degree of freedom. Experiments show that this is indeed the dominant speed in porous aggregates. However, our results show that the sound speed should be a very steep function of density once a significant number of monomers have 3 or more contacts with their neighbors. It is to be expected that the SPH approaches to modeling the properties of dust aggregates (Sirono 2004; Schäfer et al. 2007) will fail if these effects are properly not taken into account.

In summary, we conclude that we do now have a working model of dust aggregates that can be applied in parameter studies.

Acknowledgements. This work was supported by the Nederlandse Organisatie voor Wetenschapelijk Onderzoek, Grant 614.000.309. We thank Jürgen Blum for useful discussions and hospitality during several visits. We also acknowledge the financial support of Leids Kerkhoven-Bosscha Fonds.

\section{References}

Blum, J., \& Schräpler, R. 2004, Phys. Rev. Lett., 93, 115503

Blum, J., \& Wurm, G. 2000, Icarus, 143, 138

Blum, J., Wurm, G., Kempf, S., et al. 2000, Phys. Rev. Lett., 85, 2426

Blum, J., Schräpler, R., Davidsson, B. J. R., \& Trigo-Rodríguez, J. M. 2006, ApJ, 652, 1768

Boley, A. C., Mejía, A. C., Durisen, R. H., et al. 2006, ApJ, 651, 517

Boley, A. C., Hartquist, T. W., Durisen, R. H., \& Michael, S. 2007, ApJ, 656, L89

Boss, A. P. 2007, ApJ, 661, L73

Brown, R. 1828, Philos. Mag., 4, 161

Callister, Jr., W. D. 2000, Materials Science and Engineering: an Introduction, 5th edn. (Materials Science and Engineering: an Introduction, 5th edn., by W. D. Callister, Jr., 871 (Wiley-VCH), January 2006)

Chokshi, A., Tielens, A. G. G. M., \& Hollenbach, D. 1993, ApJ, 407, 806

Cuzzi, J. N., Dobrovolskis, A. R., \& Champney, J. M. 1993, Icarus, 106, 102 Dahneke, B. 1975, J. Colloid Interface Sci., 51, 58

Derjaguin, B. V., Muller, V. M., \& Toporov, Y. P. 1975, J. Colloid Interface Sci., 53,314

Dominik, C., \& Dullemond, C. P. 2008, in press

Dominik, C., \& Nübold, H. 2002, Icarus, 157, 173

Dominik, C., \& Tielens, A. G. G. M. 1995, Phil. Mag. A, 72, 783,

Dominik, C., \& Tielens, A. G. G. M. 1996, Phil. Mag. A, 73, 1279,

Dominik, C., \& Tielens, A. G. G. M. 1997, ApJ, 480, 647

Dullemond, C. P., \& Dominik, C. 2005, A\&A, 434, 971

Goldreich, P., \& Ward, W. R. 1973, ApJ, 183, 1051

Hascoët, E., Herrmann, H. J., \& Loreto, V. 1999, Phys. Rev. E, 59, 3202

Heim, L., Blum, J., Preuss, M., \& Butt, H. 1999, Phys. Rev. Lett., 83, 3328

Johansen, A., Henning, T., \& Klahr, H. 2006a, ApJ, 643, 1219

Johansen, A., Klahr, H., \& Henning, T. 2006b, ApJ, 636, 1121

Johnson, K., Kendall, K., \& Roberts, A. 1971, Proc. Roy. Soc. A, 324, 301

Kempf, S., Pfalzner, S., \& Henning, T. K. 1999, Icarus, 141, 388

Krause, M., \& Blum, J. 2004, Phys. Rev. Lett., 93, 021103

Mayer, L., Quinn, T., Wadsley, J., \& Stadel, J. 2002, Science, 298, 1756

Mayer, L., Quinn, T., Wadsley, J., \& Stadel, J. 2004, ApJ, 609, 1045

Mayer, L., Lufkin, G., Quinn, T., \& Wadsley, J. 2007, ApJ, 661, L77

Mouraille, O., Mulder, W. A., \& Luding, S. 2006, J. Stat. Mech.: Theory Exp., 7,23

Ormel, C. W., \& Cuzzi, J. N. 2007, A\&A, 466, 413

Paszun, D., \& Dominik, C. 2006, Icarus, 182, 274

Pickett, B. K., Cassen, P., Durisen, R. H., \& Link, R. 2000, ApJ, 529, 1034

Pollack, J. B., Hubickyj, O., Bodenheimer, P., et al. 1996, Icarus, 124, 62

Poppe, T., Blum, J., \& Henning, T. 2000, ApJ, 533, 454

Schäfer, C., Speith, R., \& Kley, W. 2007, A\&A, 470, 733

Sirono, S.-I. 2004, Icarus, 167, 431

Teiser, J. 2007, Untersuchungen zu präplanetarem Wachstum durch Stöı, Diplomarbeit, Technische Universität zu Braunschweig

Tsai, C., Pui, D., \& Liu, B. 1990, Aerosol Sci. Technol., 12, 497

Wada, K., Tanaka, H., Suyama, T., Kimura, H., \& Yamamoto, T. 2007, ApJ, 661, 320

Weidenschilling, S. J. 1977, MNRAS, 180, 57

Weidenschilling, S. J. 1980, Icarus, 44, 172

Weidenschilling, S. J. 1984, Icarus, 60, 553

Weidenschilling, S. J. \& Cuzzi, J. N. 1993, in Protostars and Planets III, ed. E. H. Levy, \& J. I. Lunine, 1031

Wurm, G., Paraskov, G., \& Krauss, O. 2005a, Phys. Rev. E, 71, 021304

Wurm, G., Paraskov, G., \& Krauss, O. 2005b, Icarus, 178, 253 